\title{
Respostas fisiológicas de cacharas Pseudoplatystoma reticulatum submetidos a anestésicos naturais
}

\section{Physiological responses of cacharas Pseudoplatystoma reticulatum submitted to natural anesthetics}

\author{
Milena Souza dos Santos Sanchez ${ }^{*}$; Robson Andrade Rodrigues ${ }^{2}$; \\ André Luiz Nunes ${ }^{3}$; Aline Mayra da Silva Oliveira ${ }^{4}$; Letícia Emiliani Fantini²; \\ Cristiane Meldau de Campos ${ }^{5}$
}

\begin{abstract}
Resumo
Foram avaliados tempo de indução, tempo de recuperação e respostas fisiológicas de cacharas Pseudoplatystoma reticulatum submetidos ao eugenol e mentol. Foram utilizados 42 indivíduos com peso total médio de 205,5 $\pm 59,13 \mathrm{~g}$ e comprimento total médio de $33,5 \pm 2,36 \mathrm{~cm}$, dos quais um grupo foi exposto ao eugenol $100 \mathrm{mg} \mathrm{L}^{-1}(\mathrm{n}=14)$, outro grupo ao mentol $150 \mathrm{mg} \mathrm{L}^{-1}(\mathrm{n}=14)$ e um grupo controle ( $\mathrm{n}=14)$, não exposto a nenhum anestésico. No momento 0 e $24 \mathrm{~h}$ após indução foram realizadas colheitas sanguíneas nos peixes de cada tratamento $(n=7)$ para avaliação dos parâmetros hematológicos e glicose plasmática. Os resultados foram submetidos à análise de variância $(\mathrm{P}<0,05)$ e as médias quando significativas foram comparadas pelo teste de Tukey $(\mathrm{P}<0,05)$. Ambos os anestésicos em suas respectivas concentrações induziram os peixes à anestesia cirúrgica. $\mathrm{O}$ tempo de recuperação dos animais apresentou redução significativa $(\mathrm{P}<0,05)$ quando expostos ao mentol $150 \mathrm{mg} \mathrm{L}^{-1}$. Houve efeito de interação entre os fatores para o parâmetro hematológico $\mathrm{VCM}$, visto que os peixes anestesiados com eugenol apresentaram redução significativa $(\mathrm{P}<0,05)$ do mesmo 24 h após anestesia. Os valores de glicose plasmática não diferiram entre si $0 \mathrm{~h}$ após indução, sendo que $24 \mathrm{~h}$ depois, tanto os peixes do grupo controle quanto os expostos ao eugenol e mentol apresentaram redução significativa $(\mathrm{P}<0,01)$ dos níveis plasmáticos. O eugenol a $100 \mathrm{mg} \mathrm{L}^{-1}$ e o mentol a $150 \mathrm{mg} \mathrm{L}^{-1}$ podem ser utilizados seguramente na indução anestésica de cacharas durante a realização de biometrias.
\end{abstract}

Palavras-chave: Anestesia, biometria, estresse, hematologia, peixe

\begin{abstract}
The present study the aimed to evaluate the induction time, recovery time and physiological responses of cacharas, Pseudoplatystoma reticulatum, submitted to eugenol and menthol. 42 cacharas were exposed to eugenol $100 \mathrm{mgL}^{-1}(\mathrm{n}=14)$ and menthol $150 \mathrm{mgL}^{-1}(\mathrm{n}=14)$ and the control group $(\mathrm{n}=14)$ which were not exposed to any anesthetics. At the time 0 and 24 hours after induction, the bloods of 7 fishes per treatment were collected to measure the hematologic parameters and plasma glucose
\end{abstract}

${ }^{1}$ Zootecnista, Discente do Curso de Mestrado em Zootecnia, Universidade Federal da Grande Dourados, UFGD, Dourados, MS. E-mail: milenasanchezzoo@hotmail.com

${ }^{2}$ Zootecnistas, Discente do Curso de Mestrado em Zootecnia, Universidade Estadual de Mato Grosso do Sul, UEMS, Aquidauana, MS.E-mail: robzootecnia@live.com; leticia.emiliani@hotmail.com

${ }^{3}$ Zootecnista, M.e em Zootecnia, UEMS, Aquidauna, MS. E-mail: andre_lnunes@hotmail.com

${ }^{4}$ Zootecnista, Discente do Curso de Doutorado em Zootecnia, Universidade Estadual de Maringá, UEM, Maringá, PR. E-mail: alimayrazoo@yahoo.com.br

${ }^{5}$ Prof $^{\mathrm{a}} \mathrm{Dr}^{\mathrm{a}}$ em Aquicultura, UEMS, Aquidauana, MS. E-mail: cmeldau@uems.br

* Autor para correspondência 
evaluation. The recovery was conducted in aquariums free of anesthetic, and induction and recovery times were monitored. The results were submitted to analysis of variance $(\mathrm{P}<0.05)$ and the means were compared by Tukey test $(\mathrm{P}<0.05)$. Both anesthetics, in respective concentrations, induced fishes to surgical anesthesia. There was no significant difference $(\mathrm{P}>0.05)$ for induction time, however the recovery time had a significant reduction $(\mathrm{P}<0.05)$ for the menthol $150 \mathrm{mgL}^{-1}$. There was no treatment effects on hematologic parameters, except for mean corpuscular volume (VCM), which was observed an interaction effect between factors, once fish anesthetized with eugenol showed significant reduction $(\mathrm{P}<0.05)$ on VCM 24 hours after anesthesia. Plasma glucose values did not differ at 0 hour after induction. 24 hours after induction, both control group and fishes exposed to eugenol and menthol were significantly reduce in VCM. Eugenol at $100 \mathrm{mgL}^{-1}$ and menthol at $150 \mathrm{mgL}^{-1}$ may be safely induced in cachara during biometrics procedures.

Key words: Anesthesia, biometrics, stress, hematology, fish

\section{Introdução}

O cachara Pseudoplatystoma reticulatum está entre os maiores peixes da família Pimelodidae, podendo ser encontrado nas principais bacias hidrográficas Sul-americanas, como Amazônica, do Prata e do Paraguai (BUITRAGO-SUÁREZ; BURR, 2007).

Considerado entre os peixes de água doce, uma das espécies de maior potencial de mercado para a aqüicultura brasileira. A produção intensiva de cachara depara-se com situações semelhantes à produção de outras espécies, onde, situações estressantes são impostas aos animais quando submetidos às práticas de manejo, as quais podem afetar o sistema fisiológico do animal, aumentando a suscetibilidade a doenças (ROSS; ROSS, 2008). Desta forma, o uso de anestésicos é imprescindível durante o manejo, tanto para facilitar quanto minimizar os efeitos estressantes dos procedimentos (HONCZARYK; INOUE, 2009; DELBON, 2010; SIMÕES et al., 2012).

No Brasil há uso indiscriminado de diversos anestésicos químicos como tricaína metano sulfato (MS 222), sulfato de quinaldina, benzocaína e fenoxietanol. Estes anestésicos podem causar efeitos colaterais nos peixes, como: perda de muco, irritação nas brânquias e olhos (INOUE; SANTOS NETO; MORAES, 2003), com isso, anestésicos naturais são utilizados na substituição a esses produtos, por serem menos residuais e agressivos.
Dentre os anestésicos naturais, o eugenol (INOUE et al., 2011) e o mentol (FAÇANHA; GOMES, 2005) têm se destacado devido ao alto grau de eficiência comprovada para algumas espécies. Além de apresentarem baixa toxicidade e serem facilmente encontrados no mercado nacional (GONÇALVES et al., 2008), esses anestésicos, caracterizam-se como agentes farmacológicos capazes de conduzir os peixes ao estado de perda parcial ou completa da percepção dos estímulos externos. Porém, exigem concentrações e tempos distintos para que animais da mesma espécie com tamanhos diferentes atinjam o estágio de anestesia desejado (ROSS; ROSS, 2008).

De acordo com Inoue et al. (2011) o uso de anestesia aparentemente minimiza o impacto de agentes estressores, entretanto, estudos tratando de respostas metabólicas em peixes tropicais expostos a diferentes anestésicos são ainda necessários para se determinar o tempo e concentração adequada para as espécies. Neste estudo, foram avaliados tempo de indução, tempo de recuperação e respostas fisiológicas de cacharas anestesiados com eugenol e mentol.

\section{Material e Métodos}

$\mathrm{O}$ experimento e as análises foram conduzidos, respectivamente, no Laboratório de Reprodução e Larvicultura e Laboratório de Ictioparasitologia da Universidade Estadual de Mato Grosso do Sul 
- UEMS, Unidade Universitária de Aquidauana. Cacharas, Pseudoplatystoma reticulatum, adquiridos de piscicultura comercial, foram estocados e aclimatados as condições experimentais durante 30 dias em tanques de alvenaria, com volume útil de 4.000 litros, com renovação constante de água e alimentados com ração comercial extrusada ( $40 \%$ de PB), duas vezes ao dia. Os peixes permaneceram em jejum cerca de $24 \mathrm{~h}$ antes do inicio do experimento.

Foram utilizados 42 cacharas com peso total médio de 205,5 $\pm 59,13 \mathrm{~g}$ e comprimento total médio de 33,5 $\pm 2,36 \mathrm{~cm}$, distribuídos em dois tratamentos e um grupo controle. Os tratamentos corresponderam ao eugenol na concentração de $100 \mathrm{mg} \mathrm{L}^{-1}$ e ao mentol na concentração de 150 $\mathrm{mg} \mathrm{L^{-1 }}$. A colheita de sangue foi realizada em dois momentos: $0 \mathrm{~h}$ e $24 \mathrm{~h}$ após o momento da indução, sendo que a cada colheita sanguínea sete peixes foram amostrados.

As concentrações dos anestésicos utilizadas neste experimento foram recomendadas por Nadruz (2011), que ao avaliar diversas concentrações de diferentes sedativos na indução anestésica de surubins, observou que peixes expostos aos anestésicos eugenol na concentração de $100 \mathrm{mg} \mathrm{L}^{-1} \mathrm{e}$ ao mentol na concentração de $150 \mathrm{mg} \mathrm{L}^{-1}$ não diferem entre si, quando comparados ao grupo controle.

Os banhos de imersão, uma das formas mais utilizadas no uso de anestésicos, foram realizados em aquários de vidro contendo 10 litros de água, até que os peixes atingissem o estágio de anestesia cirúrgica proposto por Ross e Ross (1999). Para determinação da influência da concentração dos anestésicos no tempo de indução e no tempo de recuperação, os animais foram expostos individualmente a cada concentração, sendo o tempo monitorado por meio de cronômetro digital até o aparecimento dos padrões comportamentais avaliados. De acordo com Park et al. (2009) os tempos de indução e recuperação devem atingir um limite de 180 s e 600 s respectivamente, para serem considerados ótimos.
Depois de anestesiados os peixes foram submetidos à colheita de sangue e biometria. Posteriormente, retornaram individualmente ao aquário livre de anestésico, para se determinar o tempo de recuperação, que consiste no retorno total do equilíbrio estabelecido por Hikasa et al. (1986). Peixes que tiveram seu sangue colhido 24 $\mathrm{h}$ após a indução anestésica, também passaram pelo procedimento de biometria e sofreram manipulação simulando a colheita sanguínea $0 \mathrm{~h}$ após a indução anestésica.

Os peixes amostrados $24 \mathrm{~h}$ após a indução anestésica foram mantidos nos tanques de alvenaria até o momento da colheita de sangue, os animais foram capturados e contidos mecanicamente com auxílio de um pano umedecido, com intuito de conter o animal e minimizar o estresse. Durante o experimento verificou-se a mortalidade dos animais e também foram monitorados oxigênio dissolvido e temperatura da água dos aquários, com oxímetro portátil YSI.

O sangue foi colhido por punção do vaso caudal, utilizando seringas e agulhas diferentes, em função do parâmetro a ser analisado. Para as análises hematológicas, o sangue foi colhido com seringas e agulhas umedecidas internamente com EDTA a $3 \%$ e para análise de glicose plasmática as seringas foram umedecidas internamente com antiglicolítico (Glistab). Foi determinado o percentual de microhematócrito (GOLDENFARB et al., 1971), hemoglobinapelo métododecianometahemoglobina (COLLIER, 1944) e a contagem de eritrócitos em câmara de Neubauer. De posse desses dados, foram calculados os índices hematimétricos (WINTROBE, 1934), compreendidos pelo volume corpuscular médio (VCM) e concentração de hemoglobina corpuscular média (CHCM). A concentração de glicose plasmática foi determinada a partir de kit comercial colorimétrico (LabTest $\AA$ ).

Para os tempos de indução e de recuperação o delineamento experimental adotado foi inteiramente casualizado com dois tratamentos 
(anestésicos) e sete repetições, sendo cada peixe considerado uma unidade experimental. Para os parâmetros hematológicos e glicose plasmática o delineamento experimental adotado foi inteiramente casualizado em esquema fatorial $3 \times 2$ (anestésicos x tempos de amostragem). Cada tratamento conteve sete repetições, sendo cada peixe considerado uma unidade experimental. Para todos os parâmetros avaliados os dados obtidos foram submetidos à análise de variância $(\mathrm{P}<0,05)$ e quando significativas as médias foram comparadas pelo teste de Tukey $(\mathrm{P}<0,05)$.

\section{Resultados}

Os parâmetros de oxigênio dissolvido e temperatura aferidos durante $\mathrm{o}$ experimento mantiveram-se entre 7,05 $\pm 0,07 \mathrm{mg} \mathrm{L}^{-1}$ e 26,9 $\pm 0,14$ ${ }^{\circ} \mathrm{C}$, respectivamente. Não foi observada mortalidade dos peixes anestesiados no período de $24 \mathrm{~h}$ após a imposição dos estímulos experimentais.

As concentrações de eugenol e de mentol utilizadas nos banhos de imersão foram capazes de induzir todos os animais ao estágio de anestesia cirúrgica. No entanto os peixes apresentaram hiperatividade ao primeiro contato com a solução anestésica de eugenol, evidenciada pela euforia e movimentos natatórios acelerados no aquário, que diminuiu de acordo com a ação do anestésico.

Os peixes submetidos à indução anestésica com mentol não demonstraram reações adversas em contato com o produto, por sua vez, apresentaram os estágios iniciais de anestesia, como perda parcial de equilíbrio, perda de reações a estímulos e redução de batimentos operculares até atingir o estágio de anestesia cirúrgica.

A concentração testada de ambos os anestésicos levaram os peixes à anestesia cirúrgica, mostrandose eficientes para serem utilizados no manejo de cacharas. Entretanto, na Tabela 1, pode-se observar que os tempos de indução apresentados pelos tratamentos não diferem $(\mathrm{P}>0,05)$ estatisticamente entre si, ao contrário do tempo de recuperação, que foi superior $(\mathrm{P}<0,05)$ para o eugenol.

Tabela 1. Tempo de indução e recuperação (média \pm erro-padrão) de cacharas Pseudoplatystoma reticulatum submetidos às concentrações de eugenol e mentol

\begin{tabular}{cccc}
\hline Tratamento & N & Indução (s) & Recuperação (s) \\
\hline Eugenol 100 $\mathbf{~ m g ~ L}^{-1}$ & 7 & $113,00 \pm 19,36$ & $309,00 \pm 36,23^{\mathrm{a}}$ \\
Mentol 150 $\mathbf{~ m g ~ L}^{-1}$ & 7 & $74,86 \pm 5,11$ & $177,57 \pm 18,02^{\mathrm{b}}$ \\
\hline
\end{tabular}

Médias com letras diferentes na mesma coluna diferem estatisticamente entre si pelo teste de Tukey $(\mathrm{P}<0,05)$.

Fonte: Elaboração dos autores.

Os dados dos parâmetros hematológicos apresentados na Tabela 2, não demonstraram efeito $(\mathrm{P}>0,05)$ dos tratamentos sobre os parâmetros hematológicos como hematócrito, taxa de hemoglobina, número de eritrócitos e $\mathrm{CHCM}$. No entanto, observou-se efeito de interação $(\mathrm{P}<0,05)$ entre os fatores para os valores de VCM, sendo que os peixes anestesiados com eugenol $100 \mathrm{mg} \mathrm{L}^{-1}$ apresentaram redução do VCM 24 h após a indução.

Foi observado efeito $(\mathrm{P}<0,01)$ de tempo de amostragem para os valores de glicose plasmática. Independente do tratamento ao qual foram submetidos, os peixes apresentaram redução significativa $(\mathrm{P}<0,01)$ dos níveis plasmáticos de glicose $24 \mathrm{~h}$ após a indução como pode ser observado na Figura 1. 
Tabela 2. Parâmetros hematológicos (média \pm erro-padrão) de cacharas Pseudoplatystoma reticulatum submetidos às concentrações de eugenol e mentol.

\begin{tabular}{|c|c|c|c|c|c|}
\hline \multirow[b]{2}{*}{ Tratamento } & \multicolumn{5}{|c|}{ Parâmetros Hematológicos } \\
\hline & $\begin{array}{c}\text { Hematócrito } \\
(\%)\end{array}$ & $\begin{array}{c}\text { Taxa de } \\
\text { Hemoglobina } \\
\left(\mathrm{g} \mathrm{dL}^{-1}\right)\end{array}$ & $\begin{array}{l}\text { Número de } \\
\text { Eritrócitos } \\
\left(\mathbf{x 1 0}^{6} \mu \mathrm{L}^{1}\right) \\
\end{array}$ & $\begin{array}{c}\text { VCM } \\
\text { (fL) }\end{array}$ & $\begin{array}{c}\text { CHCM } \\
\left(\mathrm{g} \mathrm{dL}-{ }^{-1}\right)\end{array}$ \\
\hline \multicolumn{6}{|c|}{0 horas } \\
\hline Controle & $22,43 \pm 1,73$ & $4,17 \pm 0,39$ & $1,71 \pm 0,20$ & $135,11 \pm 7,41^{\mathrm{aA}}$ & $18,54 \pm 0,93$ \\
\hline Eugenol & $27,29 \pm 1,15$ & $4,95 \pm 0,38$ & $1,80 \pm 0,08$ & $151,89 \pm 5,45^{\mathrm{aA}}$ & $18,16 \pm 1,15$ \\
\hline Mentol & $22,00 \pm 1,53$ & $4,39 \pm 0,25$ & $1,76 \pm 0,14$ & $128,42 \pm 10,39^{\mathrm{aA}}$ & $21,08 \pm 2,87$ \\
\hline \multicolumn{6}{|c|}{24 horas } \\
\hline Controle & $23,29 \pm 1,29$ & $4,53 \pm 0,25$ & $1,81 \pm 0,10$ & $130,62 \pm 8,40^{\mathrm{aA}}$ & $19,81 \pm 1,64$ \\
\hline Eugenol & $23,43 \pm 1,13$ & $3,85 \pm 0,26$ & $2,08 \pm 0,11$ & $115,44 \pm 10,93^{\mathrm{aB}}$ & $16,76 \pm 1,53$ \\
\hline Mentol & $24,57 \pm 1,29$ & $4,30 \pm 0,27$ & $1,80 \pm 0,11$ & $140,20 \pm 11,72^{\mathrm{aA}}$ & $17,66 \pm 1,10$ \\
\hline
\end{tabular}

Médias seguidas com letras minúsculas na mesma coluna indicam diferença significativa pelo teste de Tukey $(\mathrm{P}<0,05)$ entre os tratamentos dentro dos tempos de amostragem; Médias seguidas com letras maiúsculas na mesma coluna indicam efeito de interação $(\mathrm{P}<0,05)$ entre os tratamentos e os tempos de amostragem; VCM = Volume Corpuscular médio; $\mathrm{CHCM}=$ concentração de Hemoglobina Corpuscular Média; Eugenol $100 \mathrm{mg} \mathrm{L}^{-1}$; Mentol $150 \mathrm{mg} \mathrm{L}^{-1}$.

Fonte: Elaboração dos autores.

Figura 1. Glicose plasmática (média \pm erro-padrão) de cacharas Pseudoplatystoma reticulatum submetidos às concentrações de eugenol e mentol. Médias seguidas de letras diferentes diferem pelo teste de Tukey $(\mathrm{P}<0,05)$.

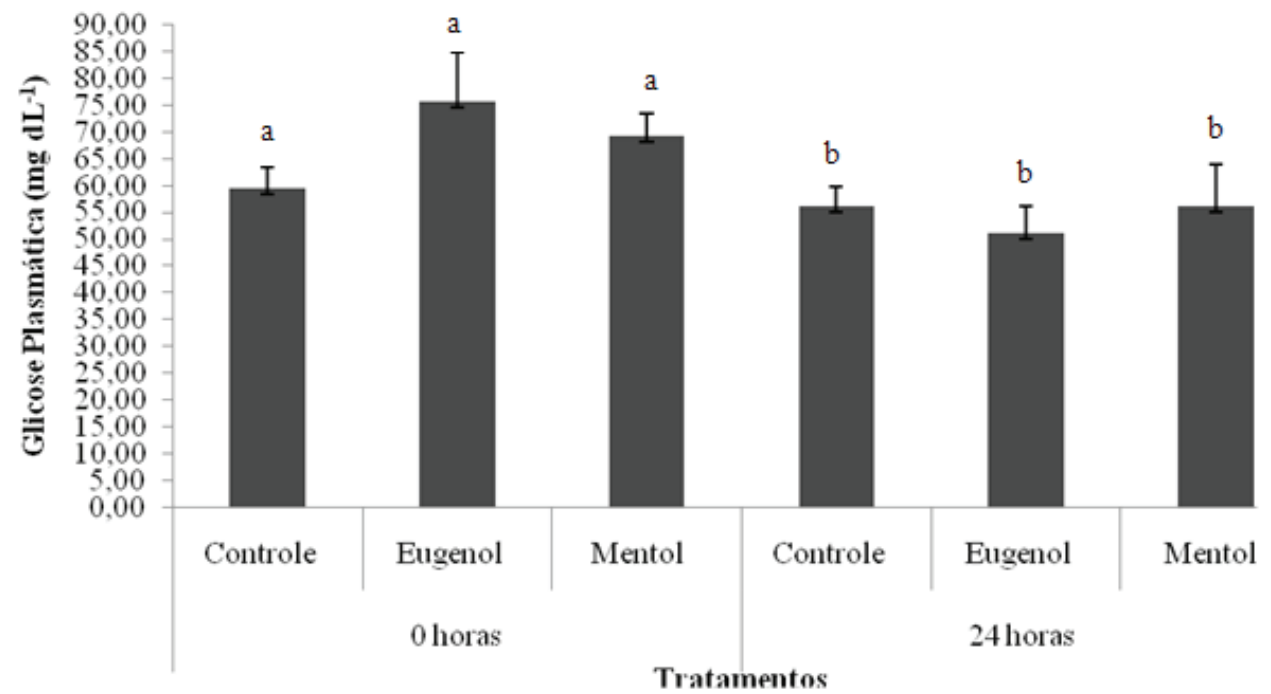

Fonte: Elaboração dos autores.

\section{Discussão}

Durante os testes de indução e recuperação dos peixes, os parâmetros de oxigênio dissolvido e temperatura aferidos foram adequados para a produção de cacharas. Desta forma, esses parâmetros não influenciaram nos resultados, sugerindo que os principais responsáveis pelo tempo necessário de anestesia cirúrgica, recuperação e posterior sobrevivência dos peixes foram os anestésicos e suas respectivas concentrações como observado por Mylonas et al. (2005).

As respostas na recuperação dos peixes não foram 
influenciadas pela colheita de sangue e biometria, porém, os peixes que tiveram seu sangue colhido 24 $\mathrm{h}$ após a indução anestésica, também passaram pelo procedimento de biometria e sofreram manipulação simulando a colheita sanguínea $0 \mathrm{~h}$ após a indução anestésica.

Vidal et al. (2006) ao utilizar eugenol na indução anestésica de juvenis de pintado Pseudoplatystoma corruscans e Vidal et al. (2007) ao submeter juvenis de matrinxã Brycon cephalus e tambaqui Colossoma macropomum a ação anestésica do eugenol, também observaram hiperatividade dos peixes, quando submetidos ao primeiro contato com a solução anestésica deste fármaco. Façanha e Gomes (2005) durante a anestesia de tambaqui com mentol e Simões e Gomes (2009) ao avaliar a eficácia do mentol como anestésico para juvenis de tilápia-do-nilo Oreochromis niloticus, assim como no presente estudo, verificaram que peixes expostos a este fármaco não demonstram reações adversas em contato com o produto, mas sim, apresentam os estágios iniciais de anestesia. Desta forma, pode-se atribuir essas reações adversas ao próprio eugenol.

Segundo Park et al. (2009), para que uma concentração anestésica seja considerada como ótima, esta deve produzir ação anestésica no período de indução de 180 s e no período de recuperação de 600 s. Portanto, pode-se observar na Tabela 1, que os tempos de indução e de recuperação obtidos no respectivo estudo não ultrapassaram os tempos supracitados, assim, pode se considerar que os anestésicos e suas respectivas dosagens são eficientes e seguros na indução anestésica de cacharas.

Simões e Gomes (2009) ao estudar a eficácia do mentol como anestésico para juvenis de tilápia-donilo verificam que entre as concentrações avaliadas $\left(50,100,150,200,250\right.$ e $\left.300 \mathrm{mg} \mathrm{L}^{-1}\right)$ a concentração de $150 \mathrm{mg} \mathrm{L}^{-1}$ de mentol é mais eficiente tanto para indução quanto para recuperação dos peixes. Resultados similares foram obtidos para tambaqui (FAÇANHA; GOMES, 2005), juvenis de pacu
Piaractus mesopotamicus (GONÇALVES et al., 2008) e dourado Salminus brasiliensis (PÁDUA et al., 2010). De forma que foram semelhantes ao do presente estudo, onde os peixes anestesiados com $150 \mathrm{mg} \mathrm{L}^{-1}$ de mentol apresentaram tempo de recuperação inferior quando comparados aos anestesiados com eugenol a $100 \mathrm{mg} \mathrm{L}^{-1}$.

Vidal et al. (2006) ao induzir juvenis de pintado com peso total médio de $27,76 \pm 7,7 \mathrm{~g}$, observam o tempo de indução de $42 \mathrm{~s}$ e tempo de recuperação de $226 \mathrm{~s}$, inferiores ao do presente estudo. Entretanto, os resultados observados pelos autores supracitados, podem ser atribuídos ao estágio de anestesia profunda avaliado ou ao peso dos peixes, uma vez que, peixes da mesma espécie de tamanhos diferentes exigem tempos distintos para que atinjam o estágio de anestesia e de recuperação desejado (ROSS; ROSS, 2008).

Nadruz (2011) observou para juvenis de cachara com peso total médio de 61,9 $\pm 13,34 \mathrm{~g} \mathrm{e}$ comprimento total médio de $22,68 \pm 1,34 \mathrm{~cm}$, que o tempo necessário para a indução de anestesia cirúrgica é $47,25 \pm 3,10 \mathrm{~s}$ e o tempo de recuperação $414,88 \pm 20,63$ s. Quando comparados ao presente estudo, o tempo de indução anestésica e recuperação foram inferiores e superiores, respectivamente, diferença que pode estar ligada ao tamanho do animal. De acordo com Roubach et al. (2002), a superfície de absorção é maior em peixes menores, os quais absorvem mais anestésicos do que os peixes maiores, e conseqüentemente, seu tempo de indução anestésica é mais rápida e sua recuperação mais lenta.

Segundo Tavares-Dias e Moraes (2004), peixes após estresse podem apresentar alterações no hemograma, ou seja, alterações nas concentrações de hemoglobina, hematócrito ou contagem de eritrócitos, indicando a ocorrência de hemoconcentração ou hemodiluição, devido à disfunção osmorregulatória. No entanto a hemoconcentração pode ser decorrente do aumento do consumo de oxigênio, na tentativa de suprir o 
aumento da demanda energética e/ou de liberação de eritrócitos pelo baço. Porém, as mudanças nas respostas hematológicas de estresse, normalmente, não são observadas em exposições agudas, mas são comuns em exposições crônicas (MORALES et al., 2005).

Nadruz (2011), ao avaliar os parâmetros hematológicos de juvenis de cachara submetidos a diferentes concentrações de eugenol $(0,25,50$, 75,100 e $125 \mathrm{mg} \mathrm{L}^{-1}$ ), verificou que os valores de VCM dos peixes expostos ao eugenol $100 \mathrm{mg}$ $\mathrm{L}^{-1}$ são significativamente inferiores aos valores encontrados para as demais concentrações, resultado este, semelhante ao encontrado no presente estudo. De acordo com o mesmo autor, ao avaliar os parâmetros eritrocitários de juvenis de surubins Pseudoplatystoma sp. submetidos a diferentes concentrações de mentol (50, 100 e 150 $\mathrm{mg} \mathrm{L}^{-1}$ ) não encontrou diferenças significativas nos valores de VCM entre os tratamentos $0 \mathrm{~h}$ após a indução anestésica. Resultado similar ao de Delbon (2010) que avaliou alterações hematológicas de tilápias anestesiadas com eugenol nos períodos subseqüentes a indução $(0,0,5,1,2,4,8,12$ e 24 h) foram encontrados no presente trabalho.

Wendelaar-Bonga (1997) explica que a resposta de estresse desencadeada por agentes estressores envolve uma série de alterações fisiológicas, classificadas como respostas primárias, secundárias e terciárias. As respostas primárias destacam-se pelo aumento na secreção de catecolaminas, adrenalina, noradrenalina e cortisol no plasma. As respostas secundárias incluem as respostas metabólicas, como a alteração na glicemia, ácido lático, glicogênio hepático e muscular e as respostas hematológicas. Sendo as respostas terciárias as alterações que levam a queda de desempenho produtivo diminuindo a resistência dos peixes a doenças.

Deriggi, Inoue e Moraes (2006) e Barbosa, Moraes e Inoue (2007) avaliaram respostas metabólicas de tilápias-do-nilo e matrinxã induzidas com o anestésico eugenol respectivamente, e observaram que os procedimentos utilizados para a indução são semelhantes aos aplicados a campo, uma vez que é realizada a transferência direta dos peixes dos tanques de estocagem para os aquários de anestesia, fato que torna estas práticas suficientes para causar estímulos adversos capazes de estimular o início das respostas ao estresse.

Uma vez iniciadas, as respostas metabólicas ao estresse surgem a partir do momento que o cérebro detecta um ou mais estímulos, quando isso acontece, dois eixos metabólicos são ativados: CPI (cérebropituitária-inter renais) e CSC (cérebro, células simpáticas cromafin) (INOUE et al., 2011). Porém, o cortisol e catecolaminas são liberados na corrente sanguínea, iniciando os processos metabólicos para a produção de energia extra o que aumenta os níveis de glicose no sangue (IWAMA et al., 2004).

No presente estudo houve redução significativa dos níveis de glicose 24 horas após a indução anestésica, resultado este, semelhante ao encontrado por Deriggi, Inoue e Moraes (2006) para tilápiasdo-nilo submetidas a diferentes concentrações de eugenol 20 e $80 \mathrm{mg} \mathrm{L}^{-1}$ avaliados 0 e $6 \mathrm{~h}$ após a indução anestésica, demonstraram níveis de glicose plasmática significativamente elevados $0 \mathrm{~h}$ após a indução. No entanto 6 h após os testes, os níveis de glicose de todos os tratamentos também reduziram, não apresentando diferença entre si.

Inoue et al. (2011), ao avaliar os níveis de glicose de tambaquis submetidos a banhos anestésicos com eugenol a concentrações de 20 e $60 \mathrm{mg} \mathrm{L}^{-1}$ por 15 minutos e amostrados 0 e $24 \mathrm{~h}$ após os banhos, puderam observar aumento significativo nos níveis de glicose $0 \mathrm{~h}$ após anestesia, sendo estes recuperados $24 \mathrm{~h}$ após os banhos. Resultados semelhantes foram encontrados por Barbosa, Moraes e Inoue (2007) para matrinxã Brycon amazonicus em banhos de eugenol (15 e $\left.60 \mathrm{mg} \mathrm{L}^{-1}\right)$ por 10 minutos, e por Simões e Gomes (2009) para juvenis de tilápia-donilo quando submetidos a concentrações de $250 \mathrm{mg}$ $\mathrm{L}^{-1}$ de mentol por 9 minutos.

Através deste exposto, e ao se comparar as 
condições do presente estudo, pode se afirmar que independente da dose do fármaco utilizado na indução anestésica, do tempo de indução e recuperação apresentado pelos peixes, o nível de glicose sempre será superior no primeiro momento, ou seja, $0 \mathrm{~h}$ após a indução anestésica, sendo estes níveis recuperados posteriormente.

De acordo com Nadruz (2011) juvenis de cachara submetidos à indução anestésica com eugenol 100 $\mathrm{mg} \mathrm{L}^{-1}$ apresentaram redução significativa para nos valores de glicose plasmática quando comparados ao grupo controle. Segundo o mesmo autor, juvenis de surubim expostos a concentração de mentol $150 \mathrm{mg}$ $\mathrm{L}^{-1}$ comparados ao grupo controle, não apresentam diferença significativa para os níveis de glicose plasmática. Assim, os resultados obtidos no presente estudo, indicam que as concentrações utilizadas de eugenol e de mentol podem ser usadas na manipulação de cacharas, uma vez que, estas não diferem entre si quando comparado ao grupo controle.

Embora os procedimentos adotados durante as fases de criação de peixes sejam considerados adversos, são inevitáveis, devido à adoção de praticas de manejo visar à otimização da cadeia produtiva. Os tratamentos avaliados neste estudo apresentaram efeito metabólico para glicose, nestas condições, considerou-se os níveis de glicose plasmática como resposta secundária ao estresse, assim, pode se afirmar que os resultados são satisfatórios para ambas as concentrações de eugenol e de mentol quando utilizados na indução anestésica de cacharas.

Os resultados aqui obtidos indicam que estes anestésicos nestas concentrações não provocam estresse de maneira adicional. Além disso, os peixes apresentam-se recuperados $24 \mathrm{~h}$ após os procedimentos experimentais.

\section{Conclusões}

O eugenol na concentração de $100 \mathrm{mg} \mathrm{L}^{-1}$ e o mentol na concentração de $150 \mathrm{mg} \mathrm{L}^{-1}$ podem ser utilizados seguramente na indução anestésica de cacharas Pseudoplatystoma reticulatum durante a realização de práticas de manejo como biometrias.

\section{Agradecimentos}

À Universidade Estadual de Mato Grosso do Sul, à empresa Mar \& Terra pelo fornecimento dos peixes e ao Macroprograma Aquabrasil com apoio financeiro da Embrapa, MPA e CNPq.

\section{Referências}

BARBOSA, L. M.; MORAES, G.; INOUE, L. A. K. A. Metabolic responses of matrinxã to eugenol in anesthetic baths. Acta Scientiarum. Biological Sciences, Maringá, v. 29, n. 3, p. 255-260, 2007.

BUITRAGO-SUÁREZ, U. A.; BURR, B. M. Taxonomy of the catfish genus Pseudoplatystoma Bleeker (Siluriformes: Pimelodidae) with recognition of eight species. Zootaxa, Auckland, n. 1512, p. 1-38, 2007.

COLLIER, H. B. The standardizations of blood haemoglobin determinations. Canadian Medical Association Journal, Vancouver, v. 50, n. 6, p. 550-552, 1944.

DELBON, M. C. Análises cromatográficas e parâmetros hematológicos de tilápia, Oreochromis niloticus, anestesiadas com eugenol em condições laboratoriais e de transporte. 2010. Tese (Doutorado em Aquicultura) Universidade Estadual Paulista Júlio de Mesquita Filho, Centro de Aqüicultura, Jaboticabal.

DERIGGI, G.; INOUE, L. A. K. A.; MORAES, G. Stress responses in nile tilapia (Oreochromis niloticus): assessment of eugenol as na alternative anesthetic. Acta Scientiarum. Biological Sciences, Maringá, v. 28, n. 3, p. 269-274, 2006.

FAÇANHA, M. F.; GOMES, L. C. A eficácia do mentol como anestésico para tambaqui (Colossoma macropomum, Characiformes: Characidae). Embrapa Amazônia Ocidental, Manaus, v. 35, n. 1, p. 71-75, 2005.

GOLDENFARB, P. B.; BOWYER, F. P.; HALL, E.; BROSIOUS, E. Reproducibility in the hematology laboratory: the microhematocrit determinations. American Journal of Clinical Pathology, Baltimore, v. 56, n. 1, p. 35-39, 1971.

GONÇALVES, A. F. N.; SANTOS, E. C. C.; FERNANDES, J. B. K.; TAKAHASHI, L. S. Mentol 
e eugenol como substitutos da benzocaína na indução anestésica de juvenis de pacu. Acta Scientiarum. Biological Sciences, Maringá, v. 30, n. 3, p. 339-344, 2008.

HIKASA, Y.; TAKASE, K.; OGASAWARA, T.; OGASAWARA, S. Anaesthesia and recovery with tricane methanesulphonate, eugenol and thiopental sodium in the carp (Cyprinus carpio). Japanese Journal of Veterinary Science, v. 48, n. 2, p. 341-351, 1986.

HONCZARYK, A.; INOUE, L. A. K. A. Anestesia do pirarucu por aspersão direta nas brânquias do eugenol em solução aquosa. Ciência Rural, Santa Maria, v. 39, n. 2, p. 577-579, 2009.

INOUE, L. A. K. A.; BOIJINK, C. L.; RIBEIRO, P. T.; SILVA, A. M. D.; AFFONSO, E. G. Avaliação de respostas metabólicas do tambaqui expostos ao eugenol em banhos anestésicos. Acta Amazônica, Rio Branco, v. 41, n. 2, p. 327-332, 2011.

INOUE, L. A. K. A.; SANTOS NETO, C.; MORAES, G. Clove oil as anaesthetic for juveniles of matrinxã Brycon cephalus (Gunther, 1869). Ciência Rural, Santa Maria, v. 33, n. 5, p. 943-947, 2003.

IWAMA, G.; AFONSO, L. O.; TODGHAM, A.; ACKERMAN, P.; NAKANO, K. Are hsps suitable for indicating stressed states in fish? The Journal of Experimental Biology, v. 204, n. 1, p. 15-19, 2004.

MORALES, A. E.; CARDENETE, G.; ABELLÁN, E.; GARCÍA-REJÓN, L. Stress-related physiological responses to handling in common dentex (Dentex dentex Linnaeus, 1758). Aquaculture Research, Oxford, v. 36, n. 1, p. 33-40, 2005.

MYLONAS, C. C.; CARDINALETTI, G.; SIGELAKI, I.; POLZONETTI-MAGNI, A. Comparative efficacy of clove oil and 2-phenoxyethanol as anesthetics in the aquaculture of European sea bass (Dicentrarchus labrax) and gilthead sea bream (Sparus aurata) at different temperatures. Aquaculture, Amsterdam, v. 246, n. 1-4, p. 467-481, 2005.

NADRUZ, J. O. Utilização de diferentes sedativos na indução anestésica de surubins Pseudoplatystoma sp. 2011. Trabalho de Conclusão de Curso (Graduação em Zootecnia) - Universidade Estadual de Mato Grosso do Sul, Aquidauana.

PÁDUA, S. B.; PIETRO, P. S.; IGLESSIAS-FILHO, P. S.; ISHIKAWA, M. M.; HISANO, H. Mentol como anestésico para dourado (Salminus brasiliensis). Boletim do Instituto de Pesca, São Paulo, v. 32, n. 2, p. 143-148, 2010.
PARK, I. S.; PARK, M. O.; HUR, J. W.; KIM, D. S.; CHANG, Y. J.; KIM, Y. J.; PARK, J. Y.; JOHNSON, S. C. Anesthetic effects of lidocaine-hydrochloride on water parameters in simulated transport experiment of juvenile winter flounder, Pleuronectes americanus. Aquaculture, Amsterdam, v. 294, n. 1-2, p. 76-79, 2009.

ROSS, L. G.; ROSS, B. Anaesthetic and sedative techniques for aquatic animals. 2. ed. Oxford: Blackwell Science, 1999. $159 \mathrm{p}$.

Anaesthetic and sedative techniques for aquatic animals. 3. ed. Oxford: Blackwell Science, 2008. $240 \mathrm{p}$.

ROUBACH, R.; GOMES, L. C.; LOURENÇO, J. N. P.; FONSECA, F. A. L.; VAL, A. L. Efficacy of eugenol as anaesthetic for tambaqui Juvenile (Colossoma macropomum). In: VAL, A. L.; ALMEIDA-VAL, V. M. F.; MCKINLEY, D. Tropical fish: news and reviews. Vancouver: International congress on biology of Fishes, 2002. p. 93-96.

SIMÕES, L. N.; GOMES, L. C. Eficácia do mentol como anestésico para juvenis de tilápia do nilo. Arquivo Brasileiro de Medicina Veterinária e Zootecnia, Belo Horizonte, v. 61, n. 3, p. 613-620, 2009.

SIMÕES, L. N.; GOMIDE, A. T. M.; ALMEIDA-VAL, V. M. F.; VAL, A. L.; GOMES, L. C. O uso do óleo de cravo como anestésico em juvenis avançados de tilápia do Nilo (Oreochromis niloticus). Acta Scientiarum. Animal Sciences, Maringá, v. 34, n. 2, p. 175-181, 2012.

TAVARES-DIAS, M.; MORAES, F. R. Hematologia de peixes teleósteos. Ribeirão Preto, 2004. 144 p.

VIDAL, L. V. O.; ALBINATI, R. C. B.; ALBINATI, A. C. L.; MECÊDO, G. R. Utilização do eugenol como anestésico para o manejo de juvenis de pintado (Pseudoplatystoma corruscans). Acta Scientiarum. Biological Sciences, Maringá, v. 28, n. 3, p. 275-279, 2006.

VIDAL, L. V. O.; FURUYA, W. M.; GRACIANO, T. S.; SCHAMBER, C. R.; SANTOS, L. D.; SOARES, C. M. Concentrações de eugenol para anestesia profunda e toxidade aguda em juvenis de piavuçu (Leporinus macrophalus). Acta Scientiarum. Biological Sciences, Maringá, v. 29, n. 4, p. 657-362, 2007.

WENDELAAR-BONGA, S. E. The stress response in fish. Physiological Review, Boston, v. 77, n. 3, p. 591625, 1997.

WINTROBE, M. M. Variation in the science and hemoglobin content of erythrocytes in the blood of various vertebrates. Folia Haematological, Philadelphia, v. 51, n. 32, p. 32-49, 1934. 
